\title{
Study on the Influence of the Center of Gravity of Fuel Cell City Bus on its Handling Characteristics
}

\author{
Yuezhen FAN*, Chuanchao DU**, Qingchun WANG*** \\ *School of Technology, Beijing Forestry University, NO.35 Qinghua East Road, Haidian District, 100083 Beijing, China, \\ E-mail: fanyuezhen@163.com \\ **School of Technology, Beijing Forestry University, NO.35 Qinghua East Road, Haidian District, 100083 Beijing, \\ China, E-mail: 782521482@qq.com \\ ***School of Technology, Beijing Forestry University, NO.35 Qinghua East Road, Haidian District, 100083 Beijing, \\ China, E-mail: wangqingchun@bjfu.edu.cn (Corresponding Author)
}

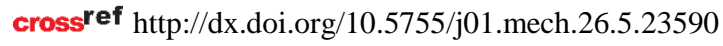

\section{Introduction}

With the rapid development of automotive industry and the application of automobile is becoming more and more popular, serious environmental pollution and resource shortage have emerged worldwide. In recent years, China has issued a series of preferential policies to support the development of products, industrialization and purchase subsidies, more fuel cells are used as power for urban buses, which has made the domestic new energy automobile industry develop rapidly $[1,2]$.

Fuel cell bus has its own particularity, high weight, battery pack weight, easy to be affected by the change of center of gravity position, especially large load transfers during acceleration and braking, which greatly affects the handling stability and driving safety of the vehicle [3].

With the change of the position of the centroid, the lateral acceleration, yaw rate and the centroid roll angle of the bus change accordingly, and the bus steering characteristic is affected. Steering characteristic is an important index for evaluating vehicle handling stability.

In 2007, Fu Jianghua, Chao kainian and Ding Jianming studied the influence of the speed, the mass and the position of the centroid on 4WS steering characteristics. The results showed that the handling stability was greatly reduced when the centroid was transferred too much [4]. In 2011, Wang Qiang and Su Xiaoping simulated and analyzed the handling stability of a bus. It showed that it was feasible to study and optimize the handling stability of fuel cell city bus by software simulation, but did not point out the relationship between the center of mass and handling stability [5]. In 2016, Cheng Haitao selected a hybrid bus in Chongqing as the research object to verify the impact of the position of the centroid on the handling stability of the hybrid bus [6]. The above research shows that the characteristics of the vehicle can be optimized theoretically by establishing vehicle dynamics and handling dynamics models and simulating them.

However, the research on handling stability is usually based on fuel vehicles, the research on fuel cell city bus is less. In addition, the performance of the bus can be directly seen through the curve of handling and stability parameters, and the parameters can be modified and the structure can be optimized easily, so as to shorten the research period and save the cost. Therefore, appropriate position of the centroid can improve the handling characteristics of the bus [7,8].

\section{Dynamics modelling}

\subsection{Dynamics model}

Assuming that the bus body is a rigid body. When a bus moves with constant speed in the horizontal plane, the bus body is connected to the wheels by suspension springs and shock absorbers, the wheels are supported on uneven road by tires with certain elasticity and damping. The bus body has three degrees of freedom: vertical displacement, rotation around the $\mathrm{X}$ and $\mathrm{Y}$ axis. The four wheels have four degrees of freedom in vertical motion. The threedimensional model has a total of 7DOF. In addition, the battery pack is also connected to the bus body through springs and dampers with suitable stiffness, which is approximately considered to be symmetrically arranged on the longitudinal axis. In discussion, this battery pack model also has three degrees of freedom. The vehicle model including battery pack is shown in Fig. 1 [9].

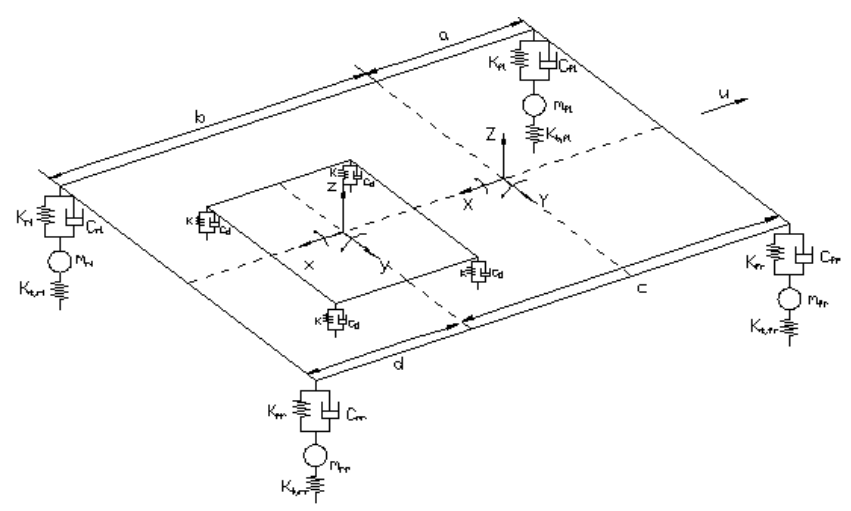

Fig. 1Vehicle dynamics mode motion is:

$$
\begin{aligned}
& I_{y c} \ddot{\theta}=\left[K_{r l}\left(z_{w, r l}-z_{r l}\right)+C_{r l}\left(\dot{z}_{w, r l}-\dot{z}_{r l}\right)+K_{r r}\left(z_{w, r r}-z_{r r}\right)+C_{r r}\left(\dot{z}_{w, r r}-\dot{z}_{r r}\right)\right] \cdot b- \\
& -\left[K_{f l}\left(z_{w, f l}-z_{f l}\right)+C_{f l}\left(\dot{z}_{w, f l}-\dot{z}_{f l}\right)+K_{f r}\left(z_{w, f r}-z_{f r}\right)+C_{f r}\left(\dot{z}_{w, f r}-\dot{z}_{f r}\right)\right] \cdot a,
\end{aligned}
$$


where: $a$ is the distance from centroid to front axle; $b$ is distance from centroid to rear axle; $\theta$ is body pitch angle; $K_{f l}, K_{f r}, K_{r l}, K_{r r}$ are the vertical stiffness of suspension spring; $z_{w, f l}, z_{w, f r}, z_{w, r l}, z_{w, r r}$ are the vertical displacement of wheels; $z_{f l}, z_{f r}, z_{r l}, z_{r r}$ are the vertical displacement of vehicle body; $C_{f l}, C_{f r}, C_{r l}, C_{r r}$ are the damping coefficient of suspension. The differential equation of the bus body rolling motion is:

$$
\begin{aligned}
& I_{x c} \ddot{\varphi}=\left[K_{f l}\left(z_{w, f l}-z_{f l}\right)+C_{f l}\left(\dot{z}_{w, f l}-\dot{z}_{f l}\right)-K_{f r}\left(z_{w, f r}-z_{f r}\right)-C_{f r}\left(\dot{z}_{w, f r}-\dot{z}_{f r}\right)\right] \cdot B_{1}+ \\
& +\left[K_{r l}\left(z_{w, r l}-z_{r l}\right)+C_{r l}\left(\dot{z}_{w, r l}-\dot{z}_{r l}\right)-K_{r r}\left(z_{w, r r}-z_{r r}\right)-C_{r r}\left(\dot{z}_{w, r r}-\dot{z}_{r r}\right)\right] \cdot B_{2},
\end{aligned}
$$

where: $B_{1}$ is the half of the front track width; $B_{2}$ is the half of the rear track width; $I_{x c}$ is the body roll moment of inertia; $\varphi$ is the body roll angle.

Four differential equations of vertical motion can be obtained from the vertical motion of four wheels:

$$
\begin{aligned}
& m_{f l} \ddot{z}_{w, f l}=K_{t, f l}\left(Q_{f l}-z_{w . f l}\right)+K_{f l}\left(z_{f l}-z_{w, f l}\right)+ \\
& +C_{f l}\left(\dot{z}_{f l}-\dot{z}_{w, f l}\right), \\
& m_{f r} \ddot{z}_{w, f r}=K_{t, f r}\left(Q_{f r}-z_{w . f r}\right)+K_{f r}\left(z_{f l}-z_{w, f r}\right)+ \\
& +C_{f r}\left(\dot{z}_{f r}-\dot{z}_{w, f r}\right), \\
& m_{r l} \ddot{z}_{w, r l}=K_{t, r l}\left(Q_{r l}-z_{w . r l}\right)+K_{r l}\left(z_{r l}-z_{w, r l}\right)+ \\
& +C_{r l}\left(\dot{z}_{r l}-\dot{z}_{w, r l}\right), \\
& m_{r r} \ddot{z}_{w, r r}=K_{t, r r}\left(Q_{r r}-z_{w . r r}\right)+K_{r r}\left(z_{r r}-z_{w, r r}\right)+ \\
& +C_{r r}\left(\dot{z}_{r r}-\dot{z}_{w, r r}\right),
\end{aligned}
$$

where: $m_{f l}, m_{f r}, m_{r l}, m_{r r}$ are the wheel quality; $K_{t, f l}, K_{t, f r}, K_{t, r l}$,
$K_{t, r r}$ are the vertical stiffness of tire; $Q_{f l}, Q_{f r}, Q_{r l}, Q_{r r}$ are the pavement input function.

\subsection{Mathematical model}

The state-space method is used to establish the mathematical model by using MATLAB. First, transforming the above equations of motion in unified matrix forms.

$$
M \ddot{Z}+C \dot{Z}+K Z=K_{t} \mathbf{Q}
$$

In the formula:

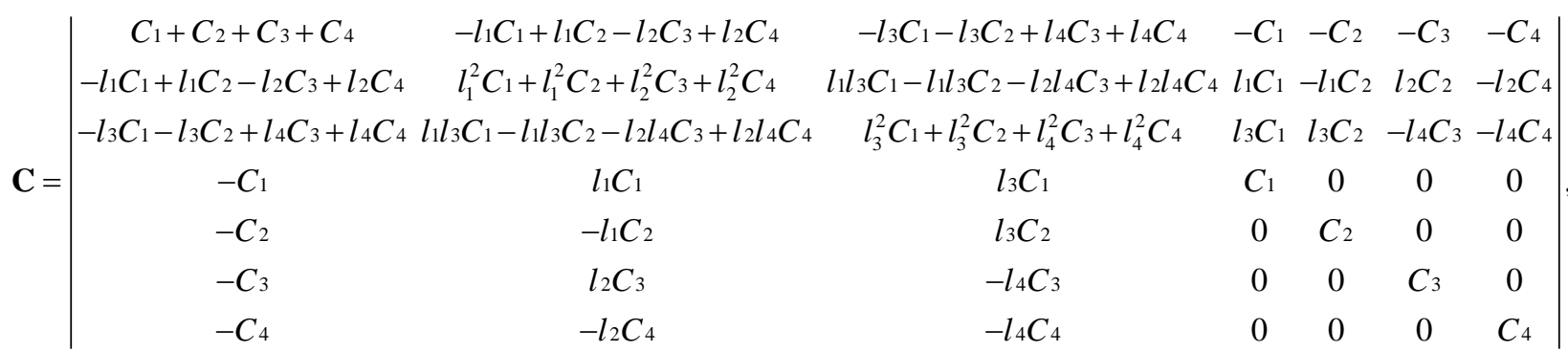

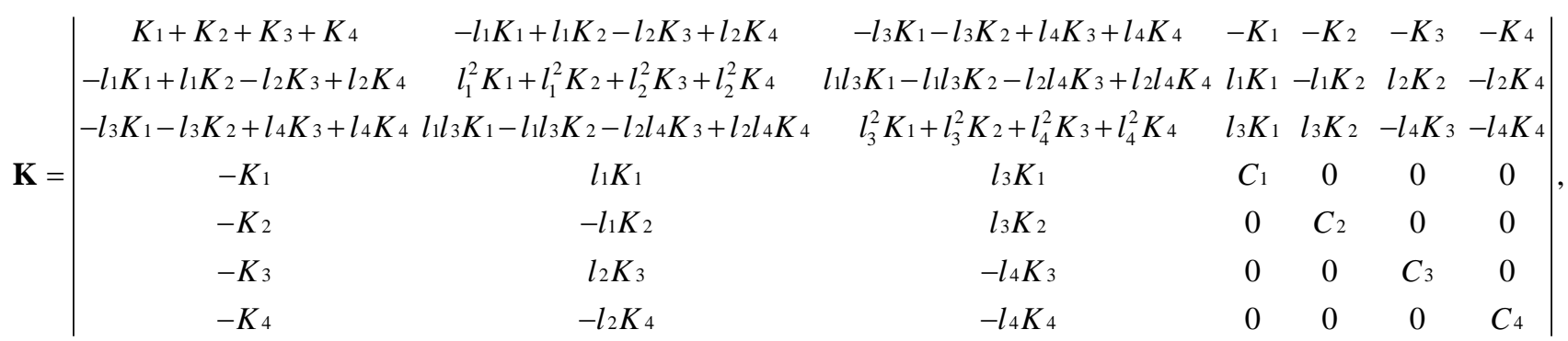

$\mathbf{K}_{\mathbf{t}}=\left|\begin{array}{cccc}0 & 0 & 0 & 0 \\ 0 & 0 & 0 & 0 \\ 0 & 0 & 0 & 0 \\ K_{t 1} & 0 & 0 & 0 \\ 0 & K_{t 2} & 0 & 0 \\ 0 & 0 & K_{t 3} & 0 \\ 0 & 0 & 0 & K_{t 4}\end{array}\right|, \quad \mathbf{Q}=\left|\begin{array}{c}Q_{1} \\ Q_{2} \\ Q_{3} \\ Q_{4}\end{array}\right|$. 
Given that in the state space, the differential equation of damping system can be written in general formula:

$$
\left[\begin{array}{cc}
\mathbf{C} & \mathbf{M} \\
\mathbf{M} & \mathbf{0}
\end{array}\right]\left(\begin{array}{c}
\dot{\mathbf{Z}} \\
\ddot{\mathbf{Z}}
\end{array}\right)+\left[\begin{array}{cc}
\mathbf{K} & \mathbf{0} \\
\mathbf{0} & -\mathbf{M}
\end{array}\right]\left(\begin{array}{l}
\mathbf{Z} \\
\dot{\mathbf{Z}}
\end{array}\right)=\left(\begin{array}{l}
\mathbf{f} \\
\mathbf{0}
\end{array}\right),
$$

$$
\text { And } \mathbf{X}=\left(\begin{array}{c}
\mathbf{Z} \\
\dot{\mathbf{Z}}
\end{array}\right), \mathbf{f}=\mathbf{K}_{\mathbf{t}} \mathbf{Q} \text { the above formula can be }
$$

written as the standard form of the state space equation:

$$
\dot{\mathbf{X}}=-\left[\begin{array}{cc}
\mathbf{C} & \mathbf{M} \\
\mathbf{M} & \mathbf{0}
\end{array}\right]^{-1}\left[\begin{array}{cc}
\mathbf{K} & \mathbf{0} \\
\mathbf{0} & -\mathbf{M}
\end{array}\right] \mathbf{X}+\left[\begin{array}{cc}
\mathbf{C} & \mathbf{M} \\
\mathbf{M} & \mathbf{0}
\end{array}\right]^{-1}\left(\begin{array}{l}
\mathbf{f} \\
\mathbf{0}
\end{array}\right) \text {. }
$$

The system output can be expressed as:

$$
\mathbf{Y}=\mathbf{C X}+\mathbf{D}
$$

In the formula:

$$
\mathbf{C}=\left[\begin{array}{llllllllllllll}
0 & 0 & 0 & 1 & 1 & 1 & 1 & 0 & 0 & 0 & 0 & 0 & 0 & 0
\end{array}\right]^{T} ; \quad \mathbf{D}=[0]
$$

\subsection{Suspension model for fixed battery pack}

The battery pack of the fuel cell city bus is also connected with the bus body through elastic suspension. Therefore, the establishment of the system model of the battery vibration can provide a reference for the design of suspension for fixed battery pack. For the movement of battery pack, the following 7 differential equations of motion can be gotten [10].

Differential equation of vertical motion:

$$
\begin{aligned}
& m \ddot{Z}=K\left(q_{f l}-d_{f l}\right)+C_{d}\left(\dot{q}_{f l}-\dot{d}_{f l}\right)+K\left(q_{f r}-d_{f r}\right)+C_{d}\left(\dot{q}_{f r}-\dot{d}_{f r}\right)+K\left(q_{r l}-d_{r l}\right)+C_{d}\left(\dot{q}_{r l}-\dot{d}_{r l}\right)+ \\
& +K\left(q_{r r}-d_{r r}\right)+C_{d}\left(\dot{q}_{r r}-\dot{d}_{r r}\right),
\end{aligned}
$$

where: $K$ is the vertical stiffness of battery pack fixture; $Z$ is the vertical displacement of battery pack; $C_{d}$ is the damping coefficient of battery pack fixture; $d_{f l}, d_{f r}, d_{r l}, d_{r r}$ are the displacement of the vertex of battery pack; $q_{f l}, q_{f r}, q_{r l}, q_{r r}$ are the fixed point input of battery pack; $m$ is the battery pack quality.

Differential equation of pitching motion:

$$
\begin{aligned}
& I_{y} \ddot{\eta}=\left[K\left(q_{r l}-d_{r l}\right)+C_{d}\left(\dot{q}_{r l}-\dot{d}_{r l}\right)+K\left(q_{r r}-d_{r r}\right)+C_{d}\left(\dot{q}_{r r}-\dot{d}_{r r}\right)\right] \cdot d- \\
& -\left[K\left(q_{f l}-d_{f l}\right)+C_{d}\left(\dot{q}_{f l}-\dot{d}_{f l}\right)+K\left(q_{f r}-d_{f r}\right)+C_{d}\left(\dot{q}_{f r}-\dot{d}_{f r}\right)\right] \cdot c,
\end{aligned}
$$

where: $c$ is the distance from battery pack centroid to front wheel; $\eta$ is the elevation angle; $I_{x}, I_{y}$ are the rotating inertia of battery pack.

Differential equation of rolling motion:

$$
\begin{aligned}
& I_{x} \ddot{\beta}=\left[K\left(q_{f l}-d_{f l}\right)+C_{d}\left(\dot{q}_{f l}-\dot{d}_{f l}\right)-K\left(q_{f r}-d_{f r}\right)-C_{d}\left(\dot{q}_{f r}-\dot{d}_{f r}\right)\right] \cdot w+ \\
& +\left[K\left(q_{r l}-d_{r l}\right)+C_{d}\left(\dot{q}_{r l}-\dot{d}_{r l}\right)+K\left(q_{r r}-d_{r r}\right)+C_{d}\left(\dot{q}_{r r}-\dot{d}_{r r}\right)\right],
\end{aligned}
$$

where: $w$ is half the width of the battery pack; $\beta$ is the roll angle of battery pack.

Vertical motion of four vertices of battery pack:

$$
\begin{aligned}
& m / 4 \cdot \dot{d}_{f l}=K\left(d_{f l}-q_{f l}\right)+C_{d}\left(\dot{d}_{f l}-\dot{q}_{f l}\right), \\
& m / 4 \cdot \ddot{d}_{f r}=K\left(d_{f r}-q_{f r}\right)+C_{d}\left(\dot{d}_{f r}-\dot{q}_{f r}\right), \\
& m / 4 \cdot \ddot{d}_{r l}=K\left(d_{r l}-q_{r l}\right)+C_{d}\left(\dot{d}_{r l}-\dot{q}_{r l}\right), \\
& m / 4 \cdot \ddot{d}_{r r}=K\left(d_{r r}-q_{r r}\right)+C_{d}\left(\dot{d}_{r r}-\dot{q}_{r r}\right)
\end{aligned}
$$

Vibration at the junction of the bus body and the battery pack is the input of the battery pack system. So, the input of the four fixed points of the battery pack is:

$$
q_{f l}=Z_{b} \cdot c / a-l_{d} / 2 \cdot \theta+w \cdot \varphi
$$

$$
\begin{aligned}
& q_{f r}=Z_{b} \cdot c / a-l_{d} / 2 \cdot \theta-w \cdot \varphi, \\
& q_{r l}=Z_{b} \cdot c / a+\frac{l}{d} / 2 \cdot \theta+w \cdot \varphi, \\
& q_{f l}=Z_{b} \cdot c / a+\frac{l_{d}}{2} / \theta \cdot \theta-w \cdot \varphi,
\end{aligned}
$$

where: $Z_{b}$ is the vertical displacement of body centroid; $I_{b}$ is the length of battery pack.

\subsection{Frequency response of the system}

According to the theoretical analysis of the above 7DOF vehicle vibration model, the amplitude-frequency characteristic curve and phase-frequency characteristic curve of the system could be obtained by using MATLAB. As shown in Fig. 2.

Usually, uneven road is the basic input of vehicle vibration, and the input frequency range is about $0.5-25 \mathrm{~Hz}$. We can know that the amplitude-frequency characteristic curve is close to the horizontal line in low-frequency area, and the amplitude ratio decreases with increasing frequency 
in Fig. 2. At a specified speed, the response bandwidth of the vehicle under no-load and full-load is nearly the same, it has good frequency response characteristic. Around $10 \mathrm{~Hz}$, which is sensitive to human, the amplitude-frequency response is close to 0 , which avoids the harm of road vibration input to human health and meets the requirement of vehicle ride comfort. It shows that the parameters are reasonable $[11,12]$.

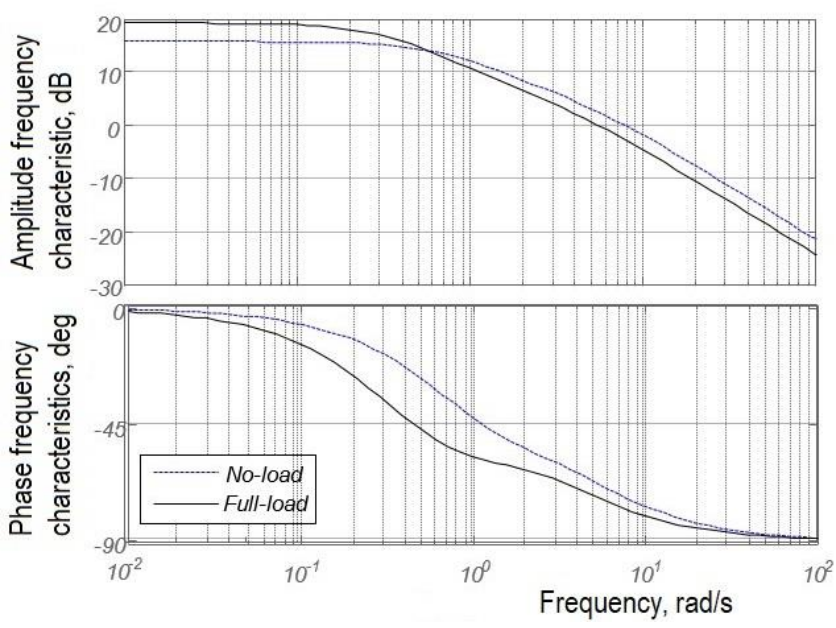

Fig. 2 System frequency response of vehicle under no-load and full-load condition

\section{Handling dynamics model}

The three degrees of freedom basic manipulation model, describing the vehicle motion, is based on the following idealized assumptions: the bus is driving on a flat road, and ignoring vertical forces and coupling effects, which is related to kinematics of driving and ignoring the air resistance, the steering system, and applying input directly to the wheels [13]. The bus is only subject to small disturbances near the equilibrium state, such as straight line driving or stable steering, and the lateral acceleration is less than $0.3 \mathrm{~g}$.

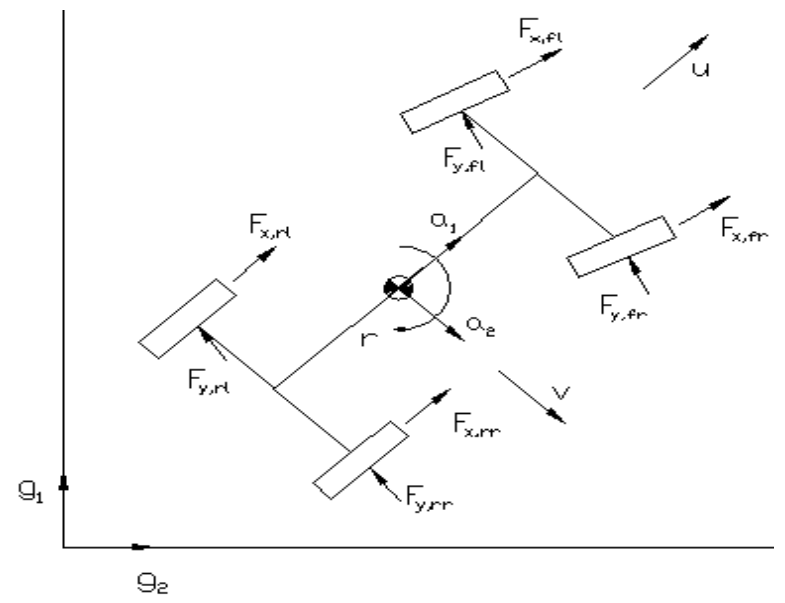

Fig. 3 Handling dynamics model

Fig. 3 shows the model of the bus. An inertial reference system $\mathrm{G}$ fixed on the ground includes inertial coordinate systems defined by three orthogonal unit vectors $\left(\mathbf{g}_{1}, \mathbf{g}_{2}, \mathbf{g}_{3}\right)$; The origin of coordinate system A, defined by three orthogonal unit vectors $\left(\mathbf{a}_{1}, \mathbf{a}_{2}, \mathbf{a}_{3}\right)$, is the intersection of the vertical line passing through the center of sprung mass and the axis of roll center. There is an angle $\Psi$ between $\mathbf{a}_{1}$ axis and $\mathbf{g}_{1}$ axis. The $\mathbf{g}_{3}$ axis and the $\mathbf{a} 3$ axis both point downward and perpendicular to the ground [14-16]. In this model, the three degrees of freedom of the bus are: moving velocity $u$, lateral velocity $v$, yaw rate $r$. The transformation relationship between coordinate axis A and coordinate axis $\mathrm{G}$ is given in Table 1, and the symbols in the graph and formula are defined in the nomenclature.

\section{Table 1}

Transformation relation between coordinate axis A and coordinate axis $\mathrm{G}$

\begin{tabular}{|c|c|c|c|}
\hline & $\mathbf{A}_{\mathbf{1}}$ & $\mathbf{A}_{\mathbf{2}}$ & $\mathbf{A}_{\mathbf{3}}$ \\
\hline $\mathbf{G}_{\mathbf{1}}$ & $\cos \Psi$ & $-\sin \Psi$ & 0 \\
\hline $\mathbf{G}_{\mathbf{2}}$ & $\sin \Psi$ & $\cos \Psi$ & 0 \\
\hline $\mathbf{G}_{\mathbf{3}}$ & 0 & 0 & 1 \\
\hline
\end{tabular}

\subsection{Front unsprung mass}

The momentum of $m_{f}$ can be expressed in coordinate system A as:

$$
\mathbf{L}_{\mathbf{f}}=m_{f} u \mathbf{a}_{\mathbf{1}}+m_{f}(v+a r) \mathbf{a}_{\mathbf{2}},
$$

where: $m_{f}$ is the front axle non-spring mass; $u$ is the forward speed; $v$ is the lateral velocity; $r$ is yaw rate.

Its angular momentum can be expressed in coordinate system A as:

$$
\mathbf{H}_{\mathbf{f}}=\left(I_{z f}+m_{f} a^{2}\right) r \mathbf{a}_{3},
$$

where: $I_{f z}$ is the yaw moment of inertia of front non-spring mass.

The change rate of momentum and angular momentum in coordinate system A can be obtained by derivation:

$$
\begin{aligned}
& \dot{\mathbf{L}}_{\mathbf{f}}=m_{f} \dot{u} a_{1}+m_{f}(\dot{v}+a \dot{r}) \mathbf{a}_{2}, \\
& \dot{\mathbf{H}}_{\mathbf{f}}=\left(I_{z f}+m_{f} a^{2}\right) \dot{r} \mathbf{a}_{3} .
\end{aligned}
$$

From the above two formulas and the transformation relationship between coordinate axis A and $\mathrm{G}$, the change rate of momentum in inertial coordinate system $\mathrm{G}$ can be expressed as:

$$
\begin{aligned}
& \frac{d \mathbf{L}_{\mathbf{f}}}{d t}=\dot{\mathbf{L}}_{\mathbf{f}}+r \mathbf{a}_{\mathbf{3}} \times \mathbf{L}_{\mathbf{f}}=m_{f}[\dot{u}-r(v+a r)] \mathbf{a}_{\mathbf{1}}+ \\
& +m_{f}(\dot{v}+u r+a \dot{r}) \mathbf{a}_{\mathbf{2}} .
\end{aligned}
$$

The change rate of angular momentum in inertial coordinate system $\mathrm{G}$ is:

$$
\frac{d \mathbf{H}_{\mathbf{f}}}{d t}=\left(I_{z f}+m_{f} a^{2}\right) \dot{r} \mathbf{a}_{\mathbf{3}}
$$

\subsection{Rear unsprung mass}

The momentum of $m_{r}$ can be expressed in coordinate system A as: 
$\mathbf{L}_{\mathbf{r}}=m_{r} u \mathbf{a}_{\mathbf{1}}+m_{r}(v-b r) \mathbf{a}_{\mathbf{2}}$,

where: $m_{r}$ is the rear axle unsprung mass.

Its angular momentum can be expressed in coordinate system A as:

$$
\mathbf{H}_{\mathbf{r}}=\left(I_{z r}+m_{r} b^{2}\right) r \mathbf{a}_{3},
$$

where: $I z r$ is the yaw moment of inertia of rear unsprung mass.

The change rate of momentum and angular momentum in coordinate system $\mathrm{A}$ can be obtained by derivation:

$$
\begin{aligned}
& \dot{\mathbf{L}}_{\mathbf{r}}=m_{r} \dot{u} \mathbf{a}_{\mathbf{1}}+m_{r}(\dot{v}-b \dot{r}) \mathbf{a}_{\mathbf{2}}, \\
& \dot{\mathbf{H}}_{\mathbf{f}}=\left(I_{z r}+m_{r} b^{2}\right) \dot{r} \mathbf{a}_{\mathbf{3}} .
\end{aligned}
$$

From the above two formulas and the transformation relationship between coordinate axis A and $\mathrm{G}$, the change rate of momentum in inertial coordinate system $\mathrm{G}$ can be expressed as:

$$
\begin{aligned}
& \frac{d \mathbf{L}_{\mathbf{r}}}{d t}=\dot{\mathbf{L}}_{\mathbf{r}}+r \mathbf{a}_{\mathbf{3}} \times \mathbf{L}_{\mathbf{r}}=m_{r}[\dot{u}-r(v-b r)] \mathbf{a}_{\mathbf{1}}+ \\
& +m_{r}(\dot{v}+u r-b \dot{r}) .
\end{aligned}
$$

The change rate of angular momentum in inertial coordinate system $\mathrm{G}$ is:

$$
\frac{d \mathbf{H}_{\mathbf{r}}}{d t}=\left(I_{z r}+m_{r} b^{2}\right) \dot{r} \mathbf{a}_{3}
$$

\subsection{Sprung mass}

As shown in Fig. 4, coordinate system B coincides with coordinate system $\mathrm{A}$ at the origin, $\mathrm{h}$ is the height of the bus body centroid from the roll axis, the transformation relationship between coordinate system $\mathrm{A}$ and coordinate system $B$ is given in Table 2 [17]

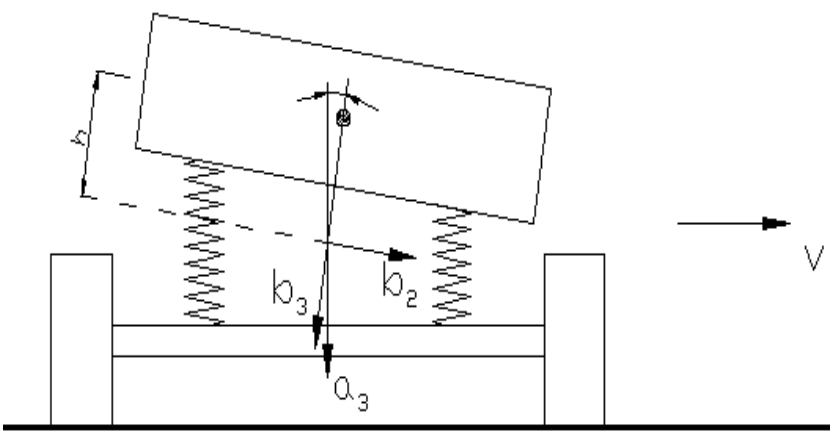

Fig. 4 Bus body roll sketch

Table 2

Transformation relation between coordinate system A and coordinate system B

\begin{tabular}{|c|c|c|c|}
\hline & $\mathbf{B}_{\mathbf{1}}$ & $\mathbf{B}_{\mathbf{2}}$ & $\mathbf{B}_{\mathbf{3}}$ \\
\hline $\mathbf{A}_{\mathbf{1}}$ & 1 & 0 & 0 \\
\hline $\mathbf{A}_{\mathbf{2}}$ & 0 & $\cos \phi$ & $-\sin \phi$ \\
\hline $\mathbf{A}_{\mathbf{3}}$ & 0 & $\sin \phi$ & $-\cos \phi$ \\
\hline
\end{tabular}

If the distance between $\mathbf{b}_{1}$ axis and bus body centroid is expressed by $h$, the position vector of the bus body centroid relative to the reference origin is:

$$
\mathbf{p}=-h \mathbf{b}_{3}=h \sin \phi \mathbf{a}_{2}-h \cos \phi \mathbf{a}_{3},
$$

where: $\phi$ is the side slope angle.

The velocity vector corresponding to the above formula is:

$$
\begin{aligned}
& \frac{d \mathbf{P}}{d t}=\dot{\mathbf{P}}+\left(r \mathbf{a}_{3}+\dot{\phi} \mathbf{b}_{1}\right) \times \mathbf{P}= \\
& =-h r \sin \phi \mathbf{a}_{1}+h \dot{\phi} \cos \phi \mathbf{a}_{2}+h \dot{\phi} \sin \phi \mathbf{a}_{3} .
\end{aligned}
$$

Combined with the velocity $\left(u \mathbf{a}_{1}+v \mathbf{a}_{2}\right)$ of the reference origin, and when $\phi$ is very small, $\sin \phi \approx 0$, it can be obtained that the momentum of the sprung mass $m_{c}$ in the reference system $\mathrm{A}$ is:

$$
\mathbf{L}_{\mathbf{c}}=m_{c} u \mathbf{a}_{\mathbf{1}}+m_{c}(v+h \dot{\phi} \cos \phi) \mathbf{a}_{\mathbf{2}},
$$

where: $m_{c}$ is the sprung mass.

The angular momentum of the bus body roll is:

$$
\mathbf{H}_{\mathbf{c 1}}=\left(I_{x c}+m_{c} h^{2}\right) \dot{\phi} \mathbf{b}_{\mathbf{1}} .
$$

The angular momentum of the bus body yaw is:

$$
\mathbf{H}_{\mathbf{c} 2}=I_{z c} r \mathbf{b}_{\mathbf{3}}=I_{z c} r\left(-\sin \phi \mathbf{a}_{\mathbf{2}}+\cos \phi \mathbf{a}_{\mathbf{3}}\right),
$$

where: $I_{z c}$ is the body yaw moment of inertia.

The change rate of momentum in inertial coordinate system $\mathrm{G}$ is:

$$
\begin{aligned}
& \frac{d \mathbf{L}_{\mathbf{c}}}{d t}=\dot{\mathbf{L}}_{\mathbf{c}}+r \mathbf{a}_{\mathbf{3}} \times \mathbf{L}_{\mathbf{c}}= \\
& =m_{c}(\dot{u}-v r) \mathbf{a}_{\mathbf{1}}+m_{c}(\dot{v}+u r+h \ddot{\phi}) \mathbf{a}_{\mathbf{2}} .
\end{aligned}
$$

The change rate of roll angular momentum in inertial reference system $\mathrm{G}$ is:

$$
\frac{d \mathbf{H}_{\mathbf{c} 1}}{d t}=\dot{\mathbf{H}}_{\mathbf{c} \mathbf{1}}+\dot{\phi} \mathbf{b}_{\mathbf{1}} \times \mathbf{H}_{\mathbf{c} 2}=\left(I_{x c}+m_{c} h^{2}\right) \ddot{\phi} \mathbf{a}_{\mathbf{1}}
$$

The change rate of yaw angular momentum in inertial coordinate system $\mathrm{G}$ is:

$$
\frac{d \mathbf{H}_{\mathrm{c} 2}}{d t}=\dot{\mathbf{H}}_{\mathbf{c} 2}+r \mathbf{a}_{\mathbf{3}} \times \mathbf{H}_{\mathbf{c} 2}==I_{z c} \dot{r} \cos \phi \mathbf{a}_{\mathbf{3}} .
$$

\subsection{Bus body roll moment}

In Fig. 5, $h$ is the distance from the bus body centroid to the roll axis, $h_{c}$ is the distance between the projection of the bus body centroid on the ground and the roll axis, $h_{c f}$ is the distance between the front wheel contact point with the ground and the roll axis, $h_{c r}$ is the distance between the rear wheel contact point with the ground and the roll axis, and the roll moment consists of the following four parts $[18,19]$. 
The first, roll moment caused by centrifugal force of sprung mass:

$$
M_{1}=-m_{c}(\dot{v}+u r) h \text {. }
$$

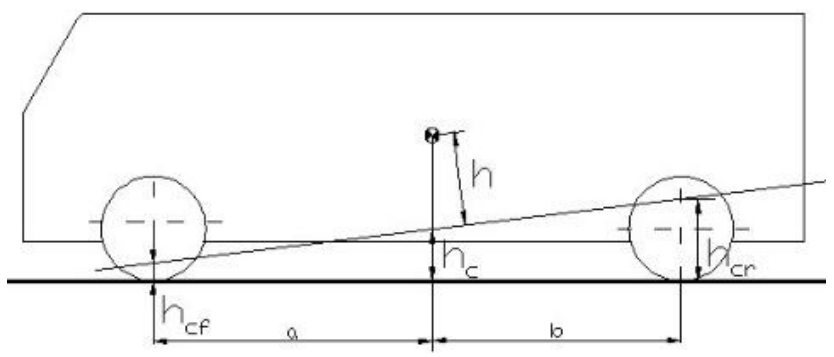

Fig. 5 Bus body roll axis

The second, roll moment caused by gravity of sprung mass after roll:

$$
M_{2}=m_{c} g h \phi \text {. }
$$

The third, roll moment caused by centrifugal force of unsprung mass:

$$
\begin{aligned}
& M_{3}=\left(h_{c f}-h_{c}\right)\left(F_{y, f l}+F_{y, f r}\right)- \\
& -\left(h_{c r}-h_{c}\right)\left(F_{y, r l}+F_{y, r r}\right) .
\end{aligned}
$$

where: $F_{y, f l}, F_{y, f r}, F_{y, r l}, F_{y, r r}$ are the force on wheel $\mathbf{a}_{2}$ direction. suspension

The forth, roll resistance moment caused by

When bus body rolls, the suspension still shows linear characteristics. Then the total roll stiffness and damping coefficient of the suspension are as follows:

$$
\begin{aligned}
& K_{\phi}=2\left(K_{f l}+K_{f r}\right) B_{1}^{2}+2\left(K_{r l}+K_{r r}\right) B_{2}^{2}, \\
& C_{\phi}=C_{f l}+C_{f r}+C_{r l}+C_{r r} .
\end{aligned}
$$

So the roll resistance moment caused by suspension is:

$$
M_{4}=-\left(K_{\phi} \phi+\frac{1}{2} C_{\phi} \dot{\phi}^{3}\right) \text {. }
$$

\subsection{Wheel cornering stiffness}

In normal state, the vertical loads on the left and right wheels of bus are generally equal, but when the bus is running on a curve, due to the action of the roll moment, the vertical loads on the left and right wheels are not equal, which will affect the stability of the bus.

As shown in Fig. 6, the gravity of the bus and the vertical reaction force on corresponding wheels are separated as a balancing force system under static condition, and the vertical ground reaction force of left and right wheels is discussed separately under the action of roll moment. Fig. 6 (a) is the force analysis diagram of the left wheel and Fig. 6 (b) is the force diagram of the right wheel.

The centrifugal force acting on bus body is distributed to the front and rear suspension roll centers according to its position of centroid. The lateral force can be expressed as:

$$
\begin{aligned}
& F_{c f}=m_{c}(\dot{v}+u r) \frac{b}{L}, \\
& F_{c r}=m_{c}(\dot{v}+u r) \frac{a}{L} .
\end{aligned}
$$
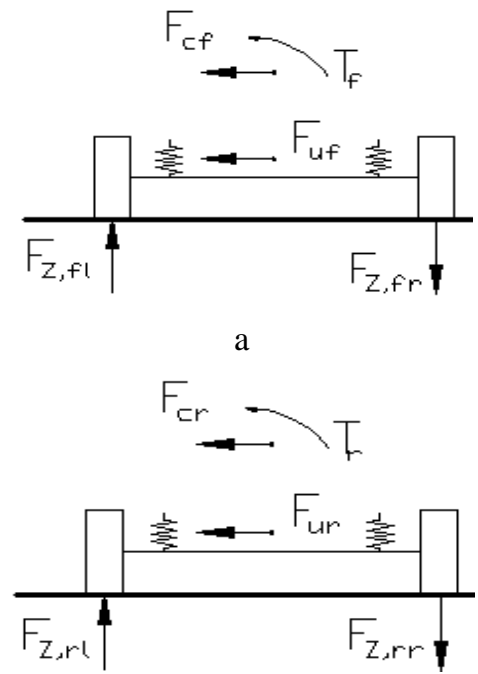

Fig. 6 Force analysis of left and right wheels: a-left wheel, b-right wheel

The roll moment of front and rear suspensions are:

$$
\begin{aligned}
& T_{\phi f}=K_{\phi f} \phi, \\
& T_{\phi r}=K_{\phi r} \phi .
\end{aligned}
$$

where: $K_{\phi f}, K_{\phi r}$ is the roll angular rigidity of front and rear suspensions.

The centrifugal force generated by the front and rear unsprung mass are:

$$
\begin{aligned}
& F_{u f}=m_{f} \frac{u^{2}}{R}, \\
& F_{u r}=m_{r} \frac{u^{2}}{R} .
\end{aligned}
$$

According to the moment balance for front axle:

$$
\begin{aligned}
& 2 F_{Z, f l} B_{1}=F_{c f}+F_{u f}+T_{f}, \\
& F_{Z, f l}=-F_{Z, f r} .
\end{aligned}
$$

where: $F_{c f}, F_{c r}$ are the distribution of body centrifugal force in front and rear suspension.

So: 


$$
\begin{aligned}
& F_{Z, f l}=\frac{1}{2 B_{1}}\left[m_{c}(\dot{v}+u r) \frac{b}{L}+m_{f} \frac{u^{2}}{R}+\right. \\
& \left.+\frac{1}{2}\left(K_{f l}+K_{f r}\right) s^{2} \phi\right], \\
& F_{Z, f r}=-\frac{1}{2 B_{1}}\left[m_{c}(\dot{v}+u r) \frac{b}{L}+m_{f} \frac{u^{2}}{R}+\right. \\
& \left.+\frac{1}{2}\left(K_{f l}+K_{f r}\right) s^{2} \phi\right] .
\end{aligned}
$$

For the rear axle:

$$
\begin{aligned}
& 2 F_{Z, r l} B_{2}=F_{c r}+F_{u r}+T_{r}, \\
& F_{Z, r l}=-F_{Z, r r} .
\end{aligned}
$$

So:

$$
\begin{aligned}
& F_{Z, r l}=\frac{1}{2 B_{2}}\left[m_{c}(\dot{v}+u r) \frac{a}{L}+m_{r} \frac{u^{2}}{R}+\right. \\
& \left.+\frac{1}{2}\left(K_{r l}+K_{r r}\right) s^{2} \phi\right], \\
& F_{Z, r r}=-\frac{1}{B_{2}}\left[m_{c}(\dot{v}+u r) \frac{a}{L}+m_{r} \frac{u^{2}}{R}+\right. \\
& \left.+\frac{1}{2}\left(K_{r l}+K_{r r}\right) s^{2} \phi\right] .
\end{aligned}
$$

The vertical reaction force, acting on wheels of the front and rear axles, is the sum of the vertical reaction force in the static state and the variation of the vertical reaction force caused by the roll. Therefore, the total vertical reaction force on the wheels are:

$$
\begin{aligned}
& N_{f l}=\frac{b}{2 L}\left(m_{f}+m_{r}+m_{c}\right)+F_{Z, f l}, \\
& N_{f r}=\frac{b}{2 L}\left(m_{f}+m_{r}+m_{c}\right)+F_{Z, f r}, \\
& N_{r l}=\frac{a}{2 L}\left(m_{f}+m_{r}+m_{c}\right)+F_{Z, r l}, \\
& N_{r r}=\frac{a}{2 L}\left(m_{f}+m_{r}+m_{c}\right)+F_{Z, r r},
\end{aligned}
$$

where: $N_{f l}, N_{f r}, N_{r l}, N_{r r}$ are the force on wheel $a_{3}$ direction.

The cornering stiffness of the tire are:

$$
\begin{aligned}
& C_{\alpha, f l}=p N_{f l}-q N_{f l}^{2}, \\
& C_{\alpha, f r}=p N_{f r}-q N_{f r}^{2}, \\
& C_{\alpha, r l}=p N_{r l}-q N_{r l}{ }^{2}, \\
& C_{\alpha, r r}=p N_{r r}-q N_{r r}{ }^{2} .
\end{aligned}
$$

where: $p, q$ is the first and second order coefficients of the slip rigidity polynomial respectively.

3.6. Wheel sideslip angle and side force

If the bus body rolls, the front steering wheel will rotate around the kingpin, and the rear wheel will rotate at a small angle around the axis perpendicular to the ground. It means the roll-steer angle also affect the steering characteristics of the bus (Fig. 7). The front wheel side-slip angle includes roll-steer angle, elastic side-slip angle and input steering angle, while the rear wheel Side-slip angle includes roll-steer angle and elastic side-slip angle.

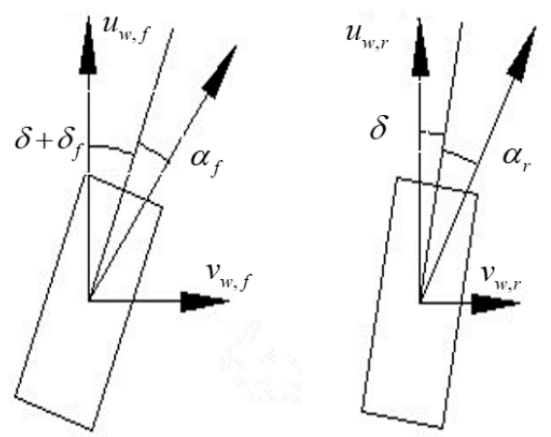

Fig. 7 Sideslip angle of front and rear wheels

The relationship between wheel roll-steer angle and bus body roll angle is:

$$
\delta=\frac{\partial \delta}{\partial \phi} \phi,
$$

where: $\frac{\partial \delta}{\partial \phi}$ is the roll steer coefficient.

As is known that the tire sideslip angle is defined as:

$$
\tan \alpha=\frac{v_{w}}{u_{w}} .
$$

The wheel speeds are:

$$
\begin{aligned}
& v_{w, f l}=v_{w, f r}=v+a r, \\
& v_{w, r l}=v_{w, r r}=v-b r, \\
& u_{w, f l}=u_{w, r l}=u+B_{1} r, \\
& u_{w, f r}=u_{w, r r}=u-B_{2} r,
\end{aligned}
$$

where: $v_{w, f l}, v_{w, f r}, v_{w, r l}, v_{w, r r}$ are the lateral velocity of wheels; $u_{w, f l}, u_{w, r l}, u_{w, f r}, u_{w, r r}$ are the forward speed of wheels.

If the front wheel inputs a steering angle $\delta_{f}$, and we define the clockwise as positive, so:

$$
\tan \alpha_{f}+\delta+\delta_{f}=\frac{v+a r}{u \pm B_{1} r}
$$


Therefore, the elastic sideslip angles of the front wheel are as follows:

$$
\begin{aligned}
& \alpha_{f l}=\tan ^{-1} \frac{v+a r}{u+B_{1} r}-\delta_{f}-\delta_{f l}, \\
& \alpha_{f r}=\tan ^{-1} \frac{v+a r}{u-B_{1} r}-\delta_{f}-\delta_{f r},
\end{aligned}
$$

where: $\delta_{f l}, \delta_{f r}, \delta_{r l}, \delta_{r r}$ are the roll steering angle;

When $\alpha$ is very small, so $\tan \alpha \approx \alpha$. When the rear axle is a non-steering axle, the elastic side-slip angle of the two rear wheels can be expressed as:

$$
\begin{aligned}
& \alpha_{r l}=\frac{v-b r}{u+B_{2} r}-\delta_{r l}, \\
& \alpha_{r r}=\frac{v-b r}{u-B_{2} r}-\delta_{r r} .
\end{aligned}
$$

Bus body roll can also lead to the change of camber angle. The relationship is as follows:

$$
\Delta \gamma=\frac{\partial \gamma}{\partial \phi} \phi
$$

where: $\gamma$ is the front wheel camber; $\Delta \gamma$ is the variation of front wheel camber.

In the formula, $\frac{\partial \gamma}{\partial \phi}$ is the change rate of camber angle caused by bus body roll. That is, the camber-roll coefficient.

Therefore, the side force which is acted on the four wheels by the ground can be expressed as:

$$
\begin{aligned}
& F_{y, f l}=C_{\alpha, f l} \alpha_{f l}+C_{\gamma, f l}(\gamma+\Delta \gamma), \\
& F_{y, f r}=C_{\alpha, f r} \alpha_{f r}+C_{\gamma, f r}(\gamma+\Delta \gamma), \\
& F_{y, r l}=C_{\alpha, r l} \alpha_{r l}, \\
& F_{y, r r}=C_{\alpha, r r} \alpha_{r r},
\end{aligned}
$$

where: $C_{\gamma, f l}, C_{\gamma, f r}, C_{\gamma, r l}, C_{\gamma, r r}$ are the extroversion stiffness of wheels.

\subsection{Motion differential equations}

According to Newton's law, the change rate of bus momentum $\mathbf{L}$ is equal to the resultant force acting on the bus, and the change rate of angular momentum $\mathbf{H}$ is equal to the resultant moment acting on the bus. The motion differential equations of the system can be obtained as follows:

$$
\begin{gathered}
\left(m_{f}+m_{r}+m_{c}\right)(\dot{u}-v r)+m_{r} b r^{2}-m_{f} a r^{2}=F_{x, f l}+F_{x, f r}+F_{x, r l}+F_{x, r r}, \\
\left(m_{f}+m_{r}+m_{c}\right)(\dot{v}+u r)+\left(a m_{f}-b m_{r}\right) \dot{r}+m_{c} h \ddot{\phi}=F_{y, f l}+F_{y, f r}+F_{y, r l}+F_{y, r r}, \\
\left(a m_{f}-b m_{r}\right)(\dot{v}+u r)+\left(I_{z c}+I_{z f}+I_{z r}+m_{f} a^{2}+m_{r} b^{2}\right) \dot{r}=a\left(F_{y, f l}+F_{y, f r}\right)-b\left(F_{y, r l}+F_{y, r r}\right), \\
\left(I_{x c}+m_{c} h^{2}\right) \ddot{\phi}=M_{1}+M_{2}+M_{3}+M_{4},
\end{gathered}
$$

where: $F_{x, f l}, F_{x, f r}, F_{x, r l}, F_{x, r r}$ are the force on wheel $\mathbf{a}_{1}$ direction; $M_{1}, M_{2}, M_{3}, M_{4}$ are the torque of body roll.

\subsection{Solving centroid location}

The change of bus centroid will cause the change of forced vibration damping ratio of the system. The change curve can be calculated by using MATLAB [20, 21]. The curve shown in Fig. 8. Abscissa $a$ represents the distance between centroid and the front axle, eta represents the damping ratio. In this curve, the abscissa of the lowest point is eta=2.87 m, and the corresponding damping ratio is $e t a=0.99$. When the value of $a$ increases or decreases, the value of eta will change accordingly.

Fig. 9 shows the yaw angular velocity step response when the vehicle damping ratio is 1.04 and the vehicle speed is greater than the critical speed $(21 \mathrm{~m} / \mathrm{s})$. The yaw angular velocity is divergent and tends to infinity gradually. It means, when eta $>1, \mathrm{~m}$, the yaw angular velocity response increases monotonously. With the increase of time, the yaw angular velocity tends to steadystate yaw angular velocity. But when the speed exceeds the critical speed, the yaw angular velocity diverges and tends to infinity. At this time, the vehicle loses stability. Normal vehicles have transient response with small damping, so the preliminary theoretical value selected of vehicle centroid is $a=2.87 \mathrm{~m}$.

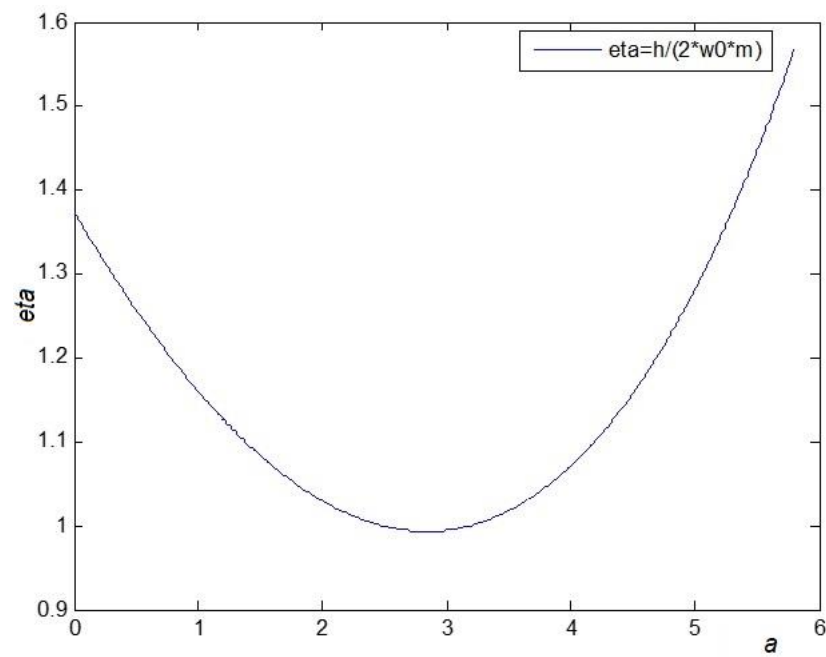

Fig. 8 The relationship between the damping ratio and vehicle centroid 


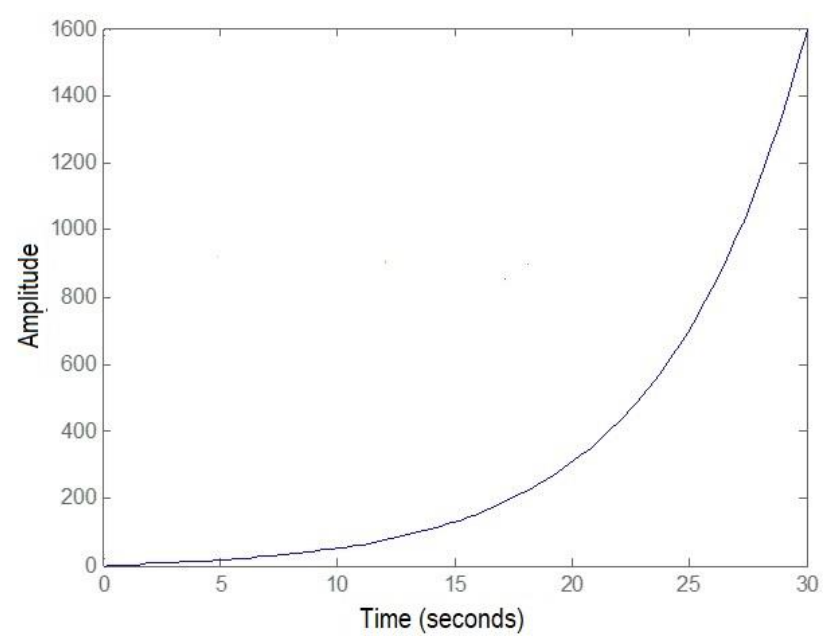

Fig. 9 Step response at eta $>1$

In order to explain the influence of the damping ratio, we can change the parameters to change the damping ratio. Vehicle centroid is moved forward or backward for a certain distance to change its damping ratio. Here, the damping ratio is taken as 1.00; By changing the parameters such as the stiffness and mass of the bus, the damping ratio changes to 0.89 . When the vehicle operates at $20 \mathrm{~m} / \mathrm{s}$, the stepped response curve of yaw angular velocity is shown in Fig. 10. We can know, when the damping ratio is 0.99 , the vehicle has the suitable steady-state response and the shortest time to reach the steady-state response, which reaches the stable value at about $3 \mathrm{~s}$, and the other two are steady after 3 seconds; When the damping ratio is 0.89 , not only the overshoot appears, but also the time required to achieve stability becomes longer; When the damping ratio is 1 , although there is no overshoot, it takes the longest time to reach stability. Based on the above analysis, it can be determined that the centroid of the fuel cell city bus should be 2.87 meters away from the front axle and 2.93 meters away from the rear axle.

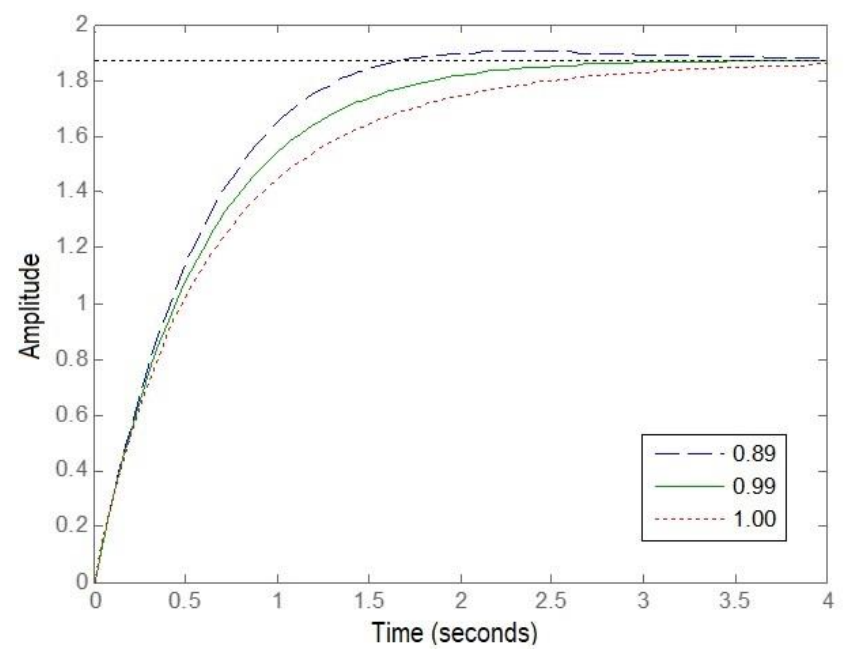

Fig. 10 Step response under different damping ratios

\section{Conclusion}

In this paper, the vehicle driving dynamics model, handling dynamics model and differential equation of motion is established and solved by using MATLAB. With uneven road as the basic input of vehicle vibration, we can obtain the amplitude-frequency characteristic curve and phase-frequency characteristic curve of the system. The theoretical solution verifies that the model data meets the requirements of vehicle ride comfort. According to the relationship between the centroid and the forced vibration damping ratio of the system, when the damping ratio is 0.99 , the vehicle has the suitable steady-state response. At this time, the vehicle centroid is 2.87 meters away from the front axle and 2.93 meters away from the rear axle. The step response of yaw angular velocity is an important index for evaluating the handling stability. It evaluates the response sensitivity of steering and the follow-up of the whole vehicle with the steering wheel.

\section{References}

1. Ning, X.; Sheng, J.; Meng, B.; et al. 2010. Vehicle suspension model and dynamic simulation on handling stability, International Conference on Mechanic Automation \& Control Engineering.

2. Wang, Q.; Oya, M.; Takagi, N.; Taira, Y.; Ota, H. 2010. Adaptive steering controller to improve handling stability for driver-combined-vehicles system, Computational Intelligence in Robotics and Automation (CIRA), 2009 IEEE International Symposium on. IEEE. http://dx.doi.org/10.1109/CIRA.2009.5423171.

3. Hu, J.; Han, Y.; Chen, J. 2017. Integrated control of direct yaw moment control and active suspension system for 4wd vehicles, Mechanics 23(3).

4. Fu, J. H.; Chao, K. N; Ding, J. M. 2007. The influence of mass center transfer on steering characteristics of four-wheel steering vehicles, Journal of Xihua University (Natural Science Edition) (04), 6 + 27-29.

5. Wang, Q.; Su X. P. 2011. Simulation and analysis of handling stability of a bus based on ADAMS / car, Mechanical design and manufacturing 11:158-160.

6. Cheng, H. T. 2016. Study on the handling stability of a hybrid bus in Chongqing, Chongqing Jiaotong University.

7. Hu, A. J.; Lv, B. Z. 2011. Development trend of automobile steering system, Henan: Henan Polytechnic University. http://dx.doi.org/10.3969/j.issn.10060006.2011.01.002.

8. Yang, W. X.; Yang, J. Z.; Zhou, Q.; Liu, J. M. 2013. Current situation and development prospects of automobile steering-by-wire system, Gansu: Lanzhou University of Technology.

http://dx.doi.org/10.3969/j.issn.10035311.2013.11.042.

9. Zong, C. F.; Guo, K. H. 2000. Research and evaluation of automobile handling and stability, Automotive technology (6): 6-11. http://dx.doi.org/10.3969/j.issn.10003703.2000.06.002.

10. Kumar, M. S.; Vijayarangan, S. 2007. Analytical and experimental studies on active suspension system of light passenger vehicle to improve ride comfort, Mechanika 3(65): 34-41.

11. Crolla, D. A.; Chen, D. C.; Whitehead, J. P.; et al. Vehicle handling assessment using a combined subjective - objective approach, SAE Paper 980226.

12. Zhao, Y. Q.; Guo, K. H. 1998. Development, research significance and basic problems of vehicle manoeuvrability evaluation, Automobile technology (5): 
$1-4$

13. Li, Z. Q.; Tang, L.; Liu, R. 2014. Dynamic Modeling Based on Zero Side-Slip Angle of the Gravity Center for Multi-Axis Steering Vehicles, Advanced Materials Research 912-914:653-657. http://dx.doi.org/10.4028/www.scientific.net/amr.912914.653.

14. Cai, Z. L. 2004. Application of dynamic simulation technology in suspension and vehicle development, Changchun: Jilin University 2004: 1-9.

15. Yin, N. D. 2001. Research on virtual test technology for handling stability of vehicle-driver-environment closed loop system, Beijing: China Agricultural University, 2001: 2-4.

16. Guo, K. H.; Ding, H. T.; Zong, C. F.; et al. 2003. Progress in the evaluation and optimization of manoeuvrability of human-vehicle closed-loop system, Journal of Mechanical Engineering 39(10): 27-34. http://dx.doi.org/10.3321/j.issn:05776686.2003.10.008.

17. Liu, M. C.; Zhang, C. N.; Wang, Z. F. 2012. Research on the influence of unsprung mass on vehicle handling stability, Advanced Materials Research 562-564:816820.

18. Reddy, R. N.; Ellis, J. R. Contribution to the simulation of driver-vehicle-road system, SAE Paper 810513.

19. Guo, K. H. 2011. Principles of vehicle manoeuvring dynamics, Nanjing: Jiangsu Science and Technology Press.

20. Adam; Toumoto. 1992. Vehicle driving characteristics, Beijing: Science Popularization Press.

21. Jin, B. Y.; Ning, G. Q.; Shi, G. B. 2010. Review of the development of automobile electric power steering system, Henan: Zhengzhou Railway Vocational and Technical College.
Yuezhen Fan, Chuanchao Du, Qingchun Wang

\section{STUDY ON THE INFLUENCE OF THE CENTER OF GRAVITY OF FUEL CELL CITY BUS ON ITS HANDLING CHARACTERISTICS}

S u m m a r y

The vehicles driven by combustion engine leads to environmental problems because of fossil fuel consumption. In recent years, many policies have adopted to support the development of new energy vehicles, especially battery electric vehicles as the main strategy in China. For the battery electric vehicles, the position of the battery pack can change the centroid position of the vehicle because of its big mass, and it can also change the loading of each tire in the motion, which has important influence on the vehicle handing and stability performance. This article studies the relationship between handling characteristic and the change of centroid position on a fuel cell city bus, and then solves the suitable centroid position of this vehicle which makes the vehicle have satisfied steering characteristic.

Keywords: centroid position, steering characteristic, modelling, simulation.

Received June 17, 2019

Accepted October 14, 2020

This article is an Open Access article distributed under the terms and conditions of the Creative Commons Attribution 4.0 (CC BY 4.0) License (http://creativecommons.org/licenses/by/4.0/). 